\title{
Multilayer Transition-Metal Dichalcogenide Channel Thin-Film Transistors
}

\author{
Eok Su Kim ${ }^{1}$, Sunkook Kim ${ }^{2}$, Yun Sung Lee ${ }^{2}$, Sang Yoon Lee ${ }^{1}$, Sunhee Lee ${ }^{1}$, Woong Choi ${ }^{3}$, \\ Hartwin Peelaers ${ }^{4}$, Chris G. Van de Walle ${ }^{4}$, Wan-Sik Hwang ${ }^{5}$, Thomas Kosel ${ }^{5}$, and Debdeep Jena ${ }^{5,+}$ \\ ${ }^{1}$ Samsung Advanced Institute of Technology, Samsung Electronics, Yongin, Gyeonggi 446-712, South Korea \\ ${ }^{2}$ Dept. of Electronics and Radio Engineering, Kyung Hee University, Yongin, Gyeonggi 446-701, South Korea \\ ${ }^{3}$ School of Advanced Materials Engineering, Kookmin University, Seoul 136-702 South Korea \\ ${ }^{4}$ Materials Department, University of California, Santa Barbara, CA 93106-5050, USA \\ ${ }^{5}$ Department of Electrical Engineering, University of Notre Dame, Notre Dame, Indiana 46556, USA
}

\section{${ }^{+}$Contact Email: djena@nd.edu}

\begin{abstract}
We show that multilayered transition-metal dichalcogenides such as multilayer $\mathrm{MoS}_{2}$ present a compelling case for Thin-Film Transistors (TFTs) for large-area display technology. Through a combined structural, optical, and electronic characterization of multilayer $\mathrm{MoS}_{2}$ TFTs, supported by density-functional theory based bandstructure calculations, we show the inherently attractive properties of these materials for such applications. We find that the current modulation of such devices is high, the current saturation is robust, normally-off operation is feasible, effective field-effect mobility at RT exceeds $100 \mathrm{~cm}^{2} / \mathrm{V} . \mathrm{s}$, and the channel can be operated in both accumulation and inversion modes. These properties make multilayer $\mathrm{MoS}_{2}$ more feasible than single layer versions to maintain processing robustness.
\end{abstract}

\section{Introduction}

Recently, interest in the electronic properties of the transition metal dichalcogenides (TMDs) of the chemical composition $\mathrm{MX}_{2}$ (where $\mathrm{M}$ : Mo, W, Ti, etc \& X: S, Se, Te, etc) was rekindled after a long hiatus by the remarkable transistor results 1 obtained with single layer $\mathrm{MoS}_{2}$. This family of layered semiconductors provides sub-nm thick $2 \mathrm{D}$ atomic crystals that represent the limits of electrostatic scaling of ultra-thin body semiconductor channels. Unlike graphene, single layer TMDs possess $\sim 1 \mathrm{eV}$ bandgaps and thus serve as channels of transistors that have high on/off ratios. The feasibility of single layer TMDs is being investigated for high-performance nanoscale digital switching transistors ${ }^{2,3}$.

Though the electronic properties of TMDs lay dormant, methods to produce multilayer structures in vast quantities have been developed since they serve as industrial scale lubricants, and in Li-ion batteries. This existing know-how can be strongly leveraged in new applications. One such area where multilayer TMDs have attractive potential is in TFTs for large area displays, where it offers a compelling mobility and subthreshold slope advantage over competing channel materials ${ }^{4}$ for low-power TFT technology [Fig 1a].

\section{Device and Crystal Structure}

The TFT test structures used to demonstrate TFT feasibility consist of exfoliated multilayer $\mathrm{MoS}_{2}$ flakes in the back-gated geometry with ALD AlOx as the gate dielectric [Fig 1b, c]. TEM imaging in the plan-view [Fig 2a] shows the lattice image [Fig 2b] and the diffraction pattern corroborates the hexagonal lattice [Fig 2c] with lattice constant $\sim 0.32 \mathrm{~nm}$. After device measurements, a few multilayer $\mathrm{MoS}_{2}$ TFTs were imaged in cross-section, which made the layered structure and the interlayer spacing $(\sim 0.62 \mathrm{~nm})$ clearly visible [Fig 2d].

\section{Bandstructure and Photocurrent Spectroscopy}

The bandstructure is of central importance in TFT design. It is well-known that standard firstprinciples density-functional theory (DFT) 
underestimates the band gap. To overcome this problem we use a hybrid functional (HSE), which adds a fraction of exact exchange and improves both band gaps and structural properties. Using this functional we calculated the band structures of single and multilayer $\mathrm{MoS}_{2}$ [Fig 3a, b]. The multilayer has an indirect bandgap; the lowestenergy direct and indirect transitions are indicated by arrows in [Fig 3b, c]. The complementary photocurrent spectrum measured on a large-area TFT shows two onsets at $\sim 1.8 \mathrm{eV}$ (indirect) and $\sim 2.0 \mathrm{eV}$ (direct). This large-area TFT exhibits desirable features with current saturation, and high on/off ratio that enabled spectral measurements at various gate voltages.

\section{Capacitance-Voltage and TFT I-V Measurements}

Capacitance-voltage (C-V) measurements of the $\mathrm{MoS}_{2}$ TFTs showed the expected accumulation capacitance for electron channel between the $\mathrm{Ti} / \mathrm{Au}$ n-type ohmic S/D contacts [Fig 5a]. However, upon slow scans, we also observed an inversion layer formation due to hole gas [Fig 5b]. The same contacts form a Schottky barrier to holes in the valence band, and thus require long RC delays for the formation of the inversion layer. The observation is corroborated by complementary I-V measurements on the same device [Fig 5b] and explained by the schematic energy band diagrams [Fig 5c]. The observation of the formation of an inversion layer is a testament to the fundamentally distinct nature of 2D crystals and layered TMDs since they have no broken bonds out of plane and lead to near-ideal band movement with remarkable immunity to Fermi level pinning. The base capacitance is that of the contact pads.

As seen in [Fig 6a], the presence of a bandgap in multilayer $\mathrm{MoS}_{2}$ makes it similar to a conventional semiconductor, and the TFT thus exhibits robust current saturation, a highly desirable property for large-area TFTs. The particular device geometry is: $t_{o x}=50 \mathrm{~nm}$, $W / L=32 / 15 \mu \mathrm{m}$, and the $t_{c h} \sim 20 \mathrm{~nm}$. The current drive is low at this stage and is severely limited by high contact resistances $(\sim 10 \quad \Omega . m m)$. Nevertheless, the current modulation is high [Fig 6b], normally-off operation feasible [Fig 6c], and the raw (no contact correction) extracted effective field-effect mobility at RT exceeds $100 \mathrm{~cm}^{2} / \mathrm{V} . \mathrm{s}$ in these multilayer $\mathrm{MoS}_{2}$ TFT test structures [Fig 6d]. Multilayer $\mathrm{MoS}_{2}$ is thus more suitable than single layer for large-area TFT applications.

\section{V.Summary}

The performance is highly encouraging, and combined with the scalability to large areas, reinforces our belief that multilayer $\mathrm{MoS}_{2}$ has immense potential for energy-efficient TFTs of the future. The technological feasibility, however, will require low-temperature processing capability, large-area scaling, flexibility (for some applications), and reliability and stability of the TFTs. Though these questions remain unanswered in this work, the initial results provide compelling motivation to pursue such studies. This project is supported by the Global Research Outreach (GRO) program of the Samsung Advanced Institute of Technology (SAIT).

\section{REFERENCES}

[1] Radisavljevic, B. et al., Nature Nanotech., 6 147 (2011).

[2] Liu, L. et al., IEEE TED 583042 (2011).

[3] Yoon, Y. et al., Nano Lett, 113768 (2011).

[4] Kamiya, T. et al., Sci. Technol. Adv. Mater., 11 044305 (2010). 

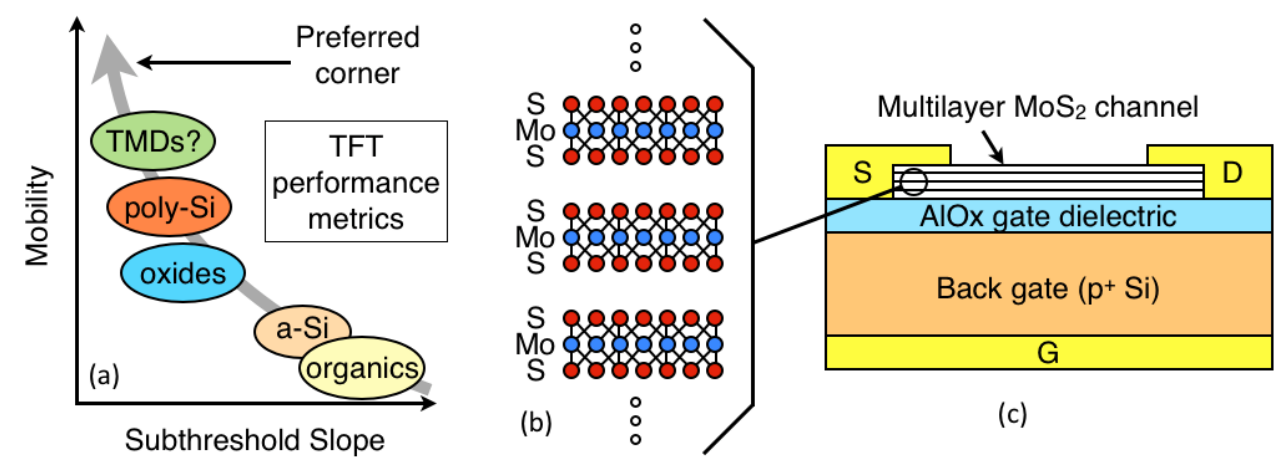

(b) $\quad:$

(c)

Figure 1: TFT material choices and desirable characteristics, and transition metal dichalcogenide (TMD) materials (e.g. $\mathrm{MoS}_{2}$ ) TFT device structure. (a) The family of materials used as channels of TFTs is shown schematically. (b and c): Multilayer $\mathrm{MoS}_{2}$ TFT test structures used in this work (c), and the layer structure of the crystal (b).
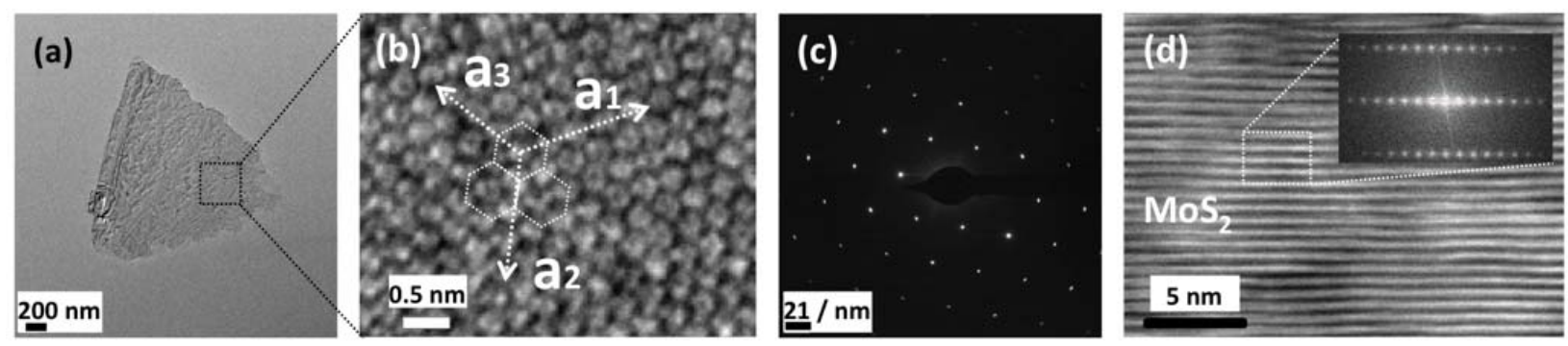

Figure 2: Crystal structure and interlayer properties of $\mathrm{MoS}_{2}$ : (a) Transmission electron micrograph (TEM) of the exfoliated $\mathrm{MoS}_{2}$ multilayers. (b) High-resolution TEM image of highly crystalline $\mathrm{MoS}_{2}$ showing a lattice constant $\mathrm{a}_{0} \sim 0.32 \mathrm{~nm}$. (c) Electron diffraction pattern showing the hexagonal symmetry, and (d) Cross-sectional TEM image of a back-gated $\mathrm{MoS}_{2}$ FET channel on a high-K dielectric, showing the multilayer structure and a Fast Fourier Transform (FFT) diffraction pattern in the insert showing an interlayer distance of $0.62 \mathrm{~nm}$.
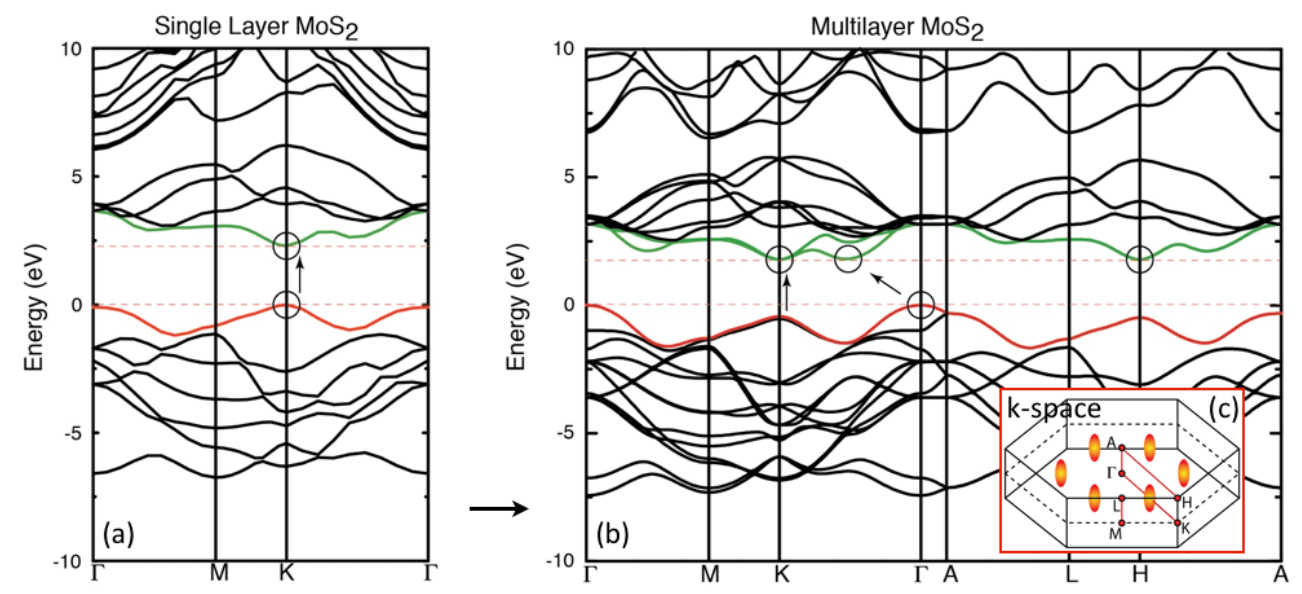

Figure 3: Energy bandstructure of (a) single layer and (b) multilayer $\mathrm{MoS}_{2}$ calculated by HSE DFT technique. Note the direct gap of single layer and indirect and direct transitions in multilayer MoS2. The inset (c) shows the k-space high symmetry points for multilayer $\mathrm{MoS}_{2}$. 

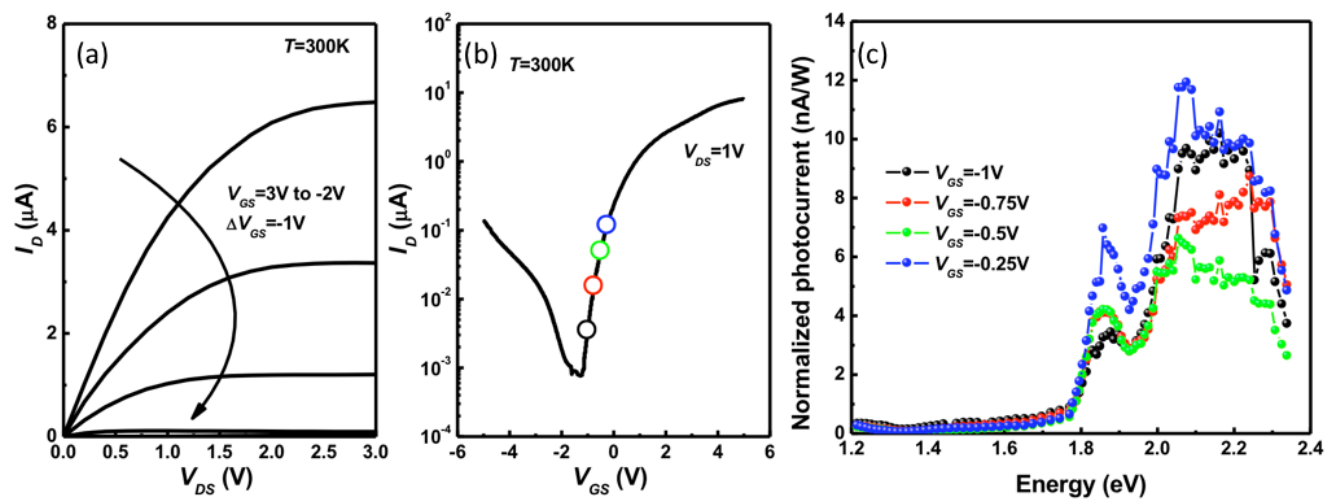

Figure 4: Direct photocurrent measurements in a back-gated multilayer $\mathrm{MoS}_{2}$ TFT. The transistor showed clear (a) current saturation and (b) gate modulation. The photocurrent spectrum (c) was obtained by biasing at various gate voltages, and indicate two distinct onsets of photocurrent at $\sim 1.8 \mathrm{eV}$ and $\sim 2.0 \mathrm{eV}$, corresponding to Fig 3(b).
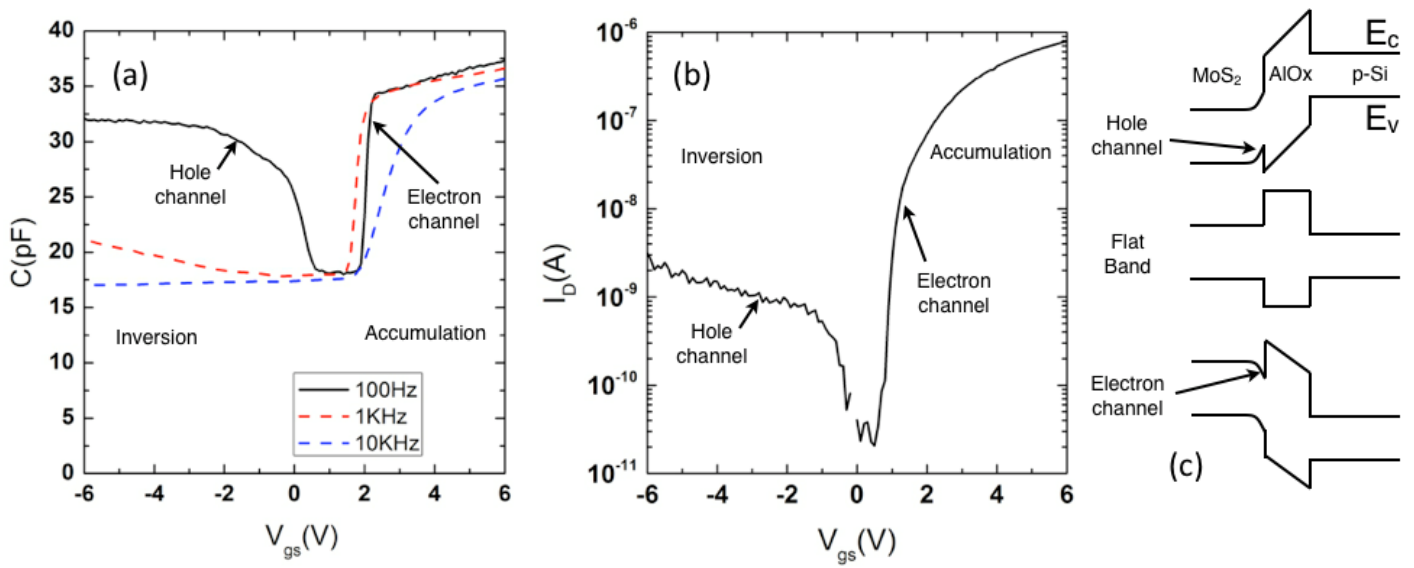

Figure 5: Capacitance-voltage measurements of $\mathrm{MoS}_{2}$ TFTs that show carrier inversion. (a) The formation of hole channel is observed only for slow scans, limited by RC charging of n-type contacts that form Schottky for holes. (b) The drain current shows clear signature of inversion and accumulation regions, and (c) Schematic energy band diagrams indicating the accumulation and inversion channels.


Figure 6: I-V characteristics and mobility of $\mathrm{MoS}_{2}$ TFT. (a) Drain current saturation is clearly observed, and (b) on/off ratios of $10^{5}$ are obtained. The transfer curve (c) allows the extraction of the 'raw' mobility values (d) that are not corrected for the contact resistance. 